Aleksandra Ochman-Gozdek, Wojciech Sarnowski and KrzyszTof Szajowski (Wrocław)

\title{
PRECISION OF SEQUENTIAL CHANGE POINT DETECTION
}

Abstract. A random sequence having two segments that are homogeneous Markov processes is registered. Each segment has its own transition probability law, and the length of the segment is unknown and random. The transition probabilities of each of the processes are known and the a priori distribution of the disorder time is given. The decision maker's aim is to detect the time when the transition probabilities change. The detection of the disorder is rarely precise. The decision maker accepts some deviation in estimation of the disorder time. In the model we consider the aim is to indicate the change point with fixed, bounded error with maximal probability. The precision differs for over and under estimation of this point. The case when the disorder does not appear with positive probability is also included. The results extend significantly the range of applications, explain the structure of optimal detector in various circumstances and show new details of the solution construction. The motivation for this investigation is the modelling of attacks in a node of networks. The objective is to detect one of the attacks immediately or in very short time before or after its appearance with the highest probability. The problem is reformulated as optimal stopping of the observed sequences. The detailed analysis of the problem is presented to show the form of the optimal decision function.

2010 Mathematics Subject Classification: Primary 60G40; Secondary 60K99, 90D60.

Key words and phrases: Bayesian approach, disorder problem, sequential detection, optimal stopping, Markov process, change point.

This paper was presented partially at the 59th ISI World Statistics Congress, 25-30 August 2013, Hong Kong, China in the session CPS018 (see [8]), and at the XXXIXth Conference on Mathematical Statistics, 2-6 December 2013, Wisła, Poland.

Received 17 September 2015; revised 11 May 2017.

Published online 15 September 2017. 
1. Introduction. Suppose that a process $X=\left\{X_{n}, n \in \mathbb{N}\right\}, \mathbb{N}=$ $\{0,1,2, \ldots\}$, is observed sequentially. It is obtained from two Markov processes by switching between them at a random time $\theta$ in such a way that the process at $\theta$ starts from the state $X_{\theta-1}$. This means that the state at time $n \in \mathbb{N}$ has a conditional distribution given the state at time $n-1$, where the formulae describing these distributions have different forms: one for $n<\theta$ and another for $n \geq \theta$. Our objective is to detect the time $\theta$ based on observation of $X$. There are some papers devoted to the discrete case of such disorder detection which generalize in various directions the basic problem stated by Shiryaev [11] (see e.g. Brodsky and Darkhovsky [5], Bojdecki [3], Bojdecki and Hosza [4], Yoshida [18], Szajowski [14, 15]).

Such a model of data appears in many practical problems of quality control (Brodsky and Darkhovsky [5], Shewhart [10] and the collection of papers [2]), traffic anomalies in networks (Dube and Mazumdar [6], Tartakovsky et al. [16]), epidemiology models (Baron [1, Siegmund [13]). The aim is to recognize the time of change from one probabilistic characteristic of the phenomenon to another.

Typically, the disorder problem is limited to the case of switching between sequences of independent random variables (see Bojdecki [3]). Some developments of the basic model can be found in Yakir [17] where the optimal rule for detecting the switching time was obtained, i.e. the time when the Markov chain of finite state-space is disordered. Moustakides [7] formulates conditions which help one to reduce the problem of the quickest detection for dependent sequences before and after the change to the case of independent random variables. Our result is a generalization of the results obtained by Bojdecki [3] and Sarnowski and Szajowski [9]. It assumes Markovian dependence structure for switched sequences (with possibly uncountable state-space). In this way we obtain an optimal rule under the probability maximizing criterion.

A formulation of the problem can be found in Section 2, The main result is presented in Section 3 .

2. Formulation of the problem. Let $(\Omega, \mathcal{F}, \mathbf{P})$ be a probability space which supports the sequence of observable random variables $\left\{X_{n}\right\}_{n \in \mathbb{N}}$ generating the filtration

$$
\mathcal{F}_{n}=\sigma\left(X_{0}, X_{1}, \ldots, X_{n}\right) .
$$

The random variables $X_{n}$ take values in $(\mathbb{E}, \mathcal{B})$, where $\mathbb{E}$ is a Borel subset of $\mathbb{R}$. On $(\mathbb{E}, \mathcal{B})$ there is a $\sigma$-additive measure $\mu$. The space $(\Omega, \mathcal{F}, \mathbf{P})$ also supports an unobservable (hence not measurable with respect to $\mathcal{F}_{n}$ ) random variable $\theta$ which has, by assumption, the zero-modified geometric distribution:

$$
\mathbf{P}(\theta=j)=\pi \mathbb{1}_{\{j=0\}}+(1-\pi) p^{j-1} q \mathbb{1}_{\{j \geq 1\}},
$$

where $q=1-p, \pi \in(0,1), j=1,2, \ldots$. 
Additionally, we introduce on $(\Omega, \mathcal{F}, \mathbf{P})$ two time-homogeneous Markov processes $\left\{X_{n}^{i}, \mathcal{G}_{n}^{i}, \mathbf{P}_{x}^{i}\right\}_{n \in \mathbb{N}}, i=0,1$, taking values in $(\mathbb{E}, \mathcal{B})$ where $\mathcal{G}_{n}^{i}$ are the smallest $\sigma$-fields for which $\left\{X_{n}^{i}\right\}, i=0,1$, are adapted, respectively. Moreover, $\left\{X_{n}^{i}\right\}_{n \in \mathbb{N}}, i=0,1$, are supposed to have transition densities with respect to $\mu$, i.e., for $i=0,1$ and $B \in \mathcal{B}$,

$$
\begin{aligned}
\mathbf{P}_{x}^{i}\left(X_{1}^{i} \in B\right) & =\mathbf{P}\left(X_{1}^{i} \in B \mid X_{0}^{i}=x\right) \\
& =\int_{B} f_{x}^{i}(y) \mu(d y)=\int_{B} \mu_{x}^{i}(d y) .
\end{aligned}
$$

The random processes $\left\{X_{n}\right\},\left\{X_{n}^{0}\right\},\left\{X_{n}^{1}\right\}$ and the random variable $\theta$ are related as follows. On the set $\theta=k$,

$$
X_{n}= \begin{cases}X_{n}^{0} & \text { if } k>n, \\ X_{n+1-k}^{1} & \text { if } k \leq n,\end{cases}
$$

where $\left\{X_{n}^{1}\right\}$ starts from $X_{k-1}^{0}$ (but is otherwise independent of $X^{0}$ ).

Let us introduce the following notation:

$$
\begin{aligned}
\underline{x}_{k, n} & =\left(x_{k}, x_{k+1}, \ldots, x_{n}\right), \quad k \leq n, \\
L_{m}\left(\underline{x}_{k, n}\right) & =\prod_{r=k+1}^{n-m} f_{x_{r-1}}^{0}\left(x_{r}\right) \prod_{r=n-m+1}^{n} f_{x_{r-1}}^{1}\left(x_{r}\right), \\
\underline{A}_{k, n} & =\underset{i=k}{\times} A_{i}=A_{k} \times A_{k+1} \times \cdots \times A_{n}, \quad A_{i} \in \mathcal{B},
\end{aligned}
$$

where the convention $\prod_{i=j_{1}}^{j_{2}} x_{i}=1$ for $j_{1}>j_{2}$ is used.

Let us now define

$$
\begin{aligned}
S_{n}\left(\underline{x}_{0, n}\right)= & \pi L_{n}\left(\underline{x}_{0, n}\right)+\bar{\pi}\left(\sum_{i=1}^{n} p^{i-1} q L_{n-i+1}\left(\underline{x}_{0, n}\right)+p^{n} L_{0}\left(\underline{x}_{0, n}\right)\right) \\
G_{l+1}\left(\underline{x}_{n-l-1, n}, \alpha\right)=\alpha L_{l+1}\left(\underline{x}_{n-l-1, n}\right) & \\
& +(1-\alpha)\left(\sum_{i=0}^{l} p^{l-i} q L_{i+1}\left(\underline{x}_{n-l-1, n}\right)+p^{l+1} L_{0}\left(\underline{x}_{n-l-1, n}\right)\right),
\end{aligned}
$$

where $x_{0}, x_{1}, \ldots, x_{n} \in \mathbb{E}^{n+1}, \alpha \in[0,1], 0 \leq n-l-1<n$, and $\bar{\pi}=1-\pi$ (cf. (2.1)).

The function $S_{n}\left(\underline{x}_{0, n}\right)$ stands for the joint density of the vector $\underline{X}_{0, n}$. For any $\underline{D}_{0, n}=\left\{\omega: \underline{X}_{0, n} \in \underline{B}_{0, n}, B_{i} \in \mathcal{B}\right\}$ and any $x \in \mathbb{E}$ we have

$$
\mathbf{P}_{x}\left(\underline{D}_{0, n}\right)=\mathbf{P}\left(\underline{D}_{0, n} \mid X_{0}=x\right)=\int_{\underline{B}_{0, n}} S_{n}\left(\underline{x}_{0, n}\right) \mu\left(d \underline{x}_{0, n}\right) .
$$

The meaning of the function $G_{n-k+1}\left(\underline{x}_{k, n}, \alpha\right)$ will be made clear below.

Overall, our model assumes that the process $\left\{X_{n}\right\}$ is obtained through switching at the random and unknown instant $\theta$ between two Markov pro- 
cesses $\left\{X_{n}^{0}\right\}$ and $\left\{X_{n}^{1}\right\}$. This means that the first observation $X_{\theta}$ after the change depends on the previous state $X_{\theta-1}$ through the transition pdf $f_{X_{\theta-1}}^{1}\left(X_{\theta}\right)$. For any fixed $d_{1}, d_{2} \in\{0,1, \ldots\}$ (the problem $\mathfrak{D}_{d_{1} d_{2}}$ ) we are looking for the stopping time $\tau^{*} \in \mathcal{T}$ such that

$$
\mathbf{P}_{x}\left(-d_{1} \leq \theta-\tau^{*} \leq d_{2}\right)=\sup _{\tau \in \mathfrak{S}^{X}} \mathbf{P}_{x}\left(-d_{1} \leq \theta-\tau \leq d_{2}\right)
$$

where $\mathfrak{S}^{X}$ denotes the set of all stopping times with respect to the filtration $\left\{\mathcal{F}_{n}\right\}_{n \in \mathbb{N}}$. Using the parameters $d_{i}, i=1,2$, we control the precision level of the detection. The problem $\mathfrak{D}_{d d}$, i.e. the case $d_{1}=d_{2}=d$, when $\pi=0$ was studied in [9].

3. Solution of the problem. Let us denote

$$
\begin{aligned}
Z_{n}^{\left(d_{1}, d_{2}\right)} & =\mathbf{P}_{x}\left(-d_{1} \leq \theta-n \leq d_{2} \mid \mathcal{F}_{n}\right), \quad n=0,1, \ldots, \\
V_{n}^{\left(d_{1}, d_{2}\right)} & =\operatorname{ess~sup}_{\left\{\tau \in \mathfrak{S}^{X}, \tau \geq n\right\}} \mathbf{P}_{x}\left(-d_{1} \leq \theta-\tau \leq d_{2} \mid \mathcal{F}_{n}\right), \quad n=0,1, \ldots, \\
\tau_{0} & =\inf \left\{n: Z_{n}^{\left(d_{1}, d_{2}\right)}=V_{n}^{\left(d_{1}, d_{2}\right)}\right\} .
\end{aligned}
$$

Notice that if $Z_{\infty}^{\left(d_{1}, d_{2}\right)}=0$, then $Z_{\tau}^{\left(d_{1}, d_{2}\right)}=\mathbf{P}_{x}\left(-d_{1} \leq \theta-\tau \leq d_{2} \mid \mathcal{F}_{\tau}\right)$ for $\tau \in \mathfrak{S}^{X}$. Since $\mathcal{F}_{n} \subseteq \mathcal{F}_{\tau}$ (when $n \leq \tau$ ), we have

$$
\begin{aligned}
V_{n}^{\left(d_{1}, d_{2}\right)} & =\underset{\tau \geq n}{\operatorname{esssup}} \mathbf{P}_{x}\left(-d_{1} \leq \theta-\tau \leq d_{2} \mid \mathcal{F}_{n}\right) \\
& =\underset{\tau \geq n}{\operatorname{esssup}} \mathbf{E}_{x}\left(\mathbb{1}_{\left\{-d_{1} \leq \theta-\tau \leq d_{2}\right\}} \mid \mathcal{F}_{n}\right)=\underset{\tau \geq n}{\operatorname{ess~sup}} \mathbf{E}_{x}\left(Z_{\tau}^{\left(d_{1}, d_{2}\right)} \mid \mathcal{F}_{n}\right) .
\end{aligned}
$$

The following lemma (see [3], 9]) ensures the existence of a solution.

Lemma 3.1. The stopping time $\tau_{0}$ defined by (3.1) is the solution of the problem 2.5).

Proof. From [3, Theorem 1] it is enough to show that $\lim _{n \rightarrow \infty} Z_{n}^{\left(d_{1}, d_{2}\right)}=0$. For all natural numbers $n, k$ with $n \geq k$, we have

$$
Z_{n}^{\left(d_{1}, d_{2}\right)}=\mathbf{E}_{x}\left(\mathbb{1}_{\left\{-d_{1} \leq \theta-n \leq d_{2}\right\}} \mid \mathcal{F}_{n}\right) \leq \mathbf{E}_{x}\left(\sup _{j \geq k} \mathbb{1}_{\left\{-d_{1} \leq \theta-j \leq d_{2}\right\}} \mid \mathcal{F}_{n}\right) .
$$

From Levy's theorem, $\lim \sup _{n \rightarrow \infty} Z_{n}^{\left(d_{1}, d_{2}\right)} \leq \mathbf{E}_{x}\left(\sup _{j \geq k} \mathbb{1}_{\left\{-d_{1} \leq \theta-j \leq d_{2}\right\}} \mid \mathcal{F}_{\infty}\right)$ where $\mathcal{F}_{\infty}=\sigma\left(\bigcup_{n=1}^{\infty} \mathcal{F}_{n}\right)$. We have $\lim \sup _{j \geq k, k \rightarrow \infty} \mathbb{1}_{\left\{-d_{1} \leq \theta-j \leq d_{2}\right\}}=0$ a.s. and by the dominated convergence theorem we get

$$
\lim _{k \rightarrow \infty} \mathbf{E}_{x}\left(\sup _{j \geq k} \mathbb{1}_{\left\{-d_{1} \leq \theta-j \leq d_{2}\right\}} \mid \mathcal{F}_{\infty}\right)=0 \quad \text { a.s. }
$$

The proof of the lemma is complete.

Through the application of the following lemma (see also [9, Lemma 3.2]) we can limit the class of possible stopping rules to $\mathfrak{S}_{d_{1}+1}^{X}$, i.e. the stopping 
times must be at least $d_{1}+1$. Then the rule $\tilde{\tau}=\max \left(\tau, d_{1}+1\right)$ is at least as good as $\tau$ :

Lemma 3.2. Let $\tau$ be a stopping rule in the problem 2.5). Then the rule $\tilde{\tau}=\max \left(\tau, d_{1}+1\right)$ is at least as good as $\tau$.

Proof. For $\tau \geq d_{1}+1$ the rules $\tau, \tilde{\tau}$ are the same. Let us consider the case when $\tau<d_{1}+1$. We have $\tilde{\tau}=d_{1}+1$, and since $\mathbf{P}_{x}(\theta \geq 1)=1$ we get

$$
\begin{aligned}
\mathbf{P}_{x}\left(-d_{1} \leq \theta-\tau \leq d_{2}\right) & =\mathbf{P}_{x}\left(\tau-d_{1} \leq \theta \leq \tau+d_{2}\right)=\mathbf{P}_{x}\left(1 \leq \theta \leq \tau+d_{2}\right) \\
\leq & \mathbf{P}_{x}\left(1 \leq \theta \leq d_{2}+d_{1}+1\right)=\mathbf{P}_{x}\left(\tilde{\tau}-d_{1} \leq \theta \leq \tilde{\tau}+d_{2}\right) \\
& =\mathbf{P}_{x}\left(-d_{1} \leq \theta-\tilde{\tau} \leq d_{2}\right) .
\end{aligned}
$$

In the case $0<\mathbf{P}_{x}\left(\tau \geq d_{1}+1\right)<1$ it is enough to replace $\tau$ by $d_{1}+1$ on $\left\{\omega: \tau \leq d_{1}\right\}$. This is the expected conclusion.

For further considerations let us define the posterior process:

$$
\begin{aligned}
& \Pi_{0}=\pi, \\
& \Pi_{n}=\mathbf{P}_{x}\left(\theta \leq n \mid \mathcal{F}_{n}\right), \quad n=1,2, \ldots,
\end{aligned}
$$

which is designed as the current information about the distribution of the disorder instant $\theta$. The next lemma transforms the payoff function to a more convenient form.

Lemma 3.3. Let

$$
h\left(\underline{x}_{0, d_{1}+1}, \alpha\right)=\left(1-p^{d_{2}}+q \sum_{m=0}^{d_{1}} \frac{L_{m+1}\left(\underline{x}_{0, d_{1}+1}\right)}{p^{m} L_{0}\left(\underline{x}_{0, d_{1}+1}\right)}\right)(1-\alpha),
$$

where $x_{0}, \ldots, x_{d_{1}+1} \in \mathbb{E}$ and $\alpha \in(0,1)$. Then

$$
\mathbf{P}_{x}\left(-d_{1} \leq \theta-n \leq d_{2}\right)=\mathbf{E}_{x}\left[h\left(\underline{X}_{n-1-d_{1}, n}, \Pi_{n}\right)\right] .
$$

Proof. We rewrite the initial criterion as the expectation

$$
\begin{aligned}
\mathbf{P}_{x}\left(-d_{1} \leq \theta-n\right. & \left.\leq d_{2}\right)=\mathbf{E}_{x}\left[\mathbf{P}_{x}\left(-d_{1} \leq \theta-n \leq d_{2} \mid \mathcal{F}_{n}\right)\right] \\
& =\mathbf{E}_{x}\left[\mathbf{P}_{x}\left(\theta \leq n+d_{2} \mid \mathcal{F}_{n}\right)-\mathbf{P}_{x}\left(\theta \leq n-d_{1}-1 \mid \mathcal{F}_{n}\right)\right] .
\end{aligned}
$$

The probabilities under the expectation can be transformed to the convenient form using Lemmata A.1, A.4 and A.5 of the Appendix (cf. [9, A1 and A4]). Next, by [9, Lemma A5] (setting $\left.l=d_{1}\right)$ we can express $\mathbf{P}_{x}\left(\theta \leq n+d_{2} \mid \mathcal{F}_{n}\right)$ in terms of $\Pi_{n}$. Straightforward calculations imply that

$$
\mathbf{P}_{x}\left(-d_{1} \leq \theta-n \leq d_{2} \mid \mathcal{F}_{n}\right)=\left(1-p^{d_{2}}+q \sum_{m=0}^{d_{1}} \frac{L_{m+1}\left(\underline{X}_{n-d_{1}-1, n}\right)}{p^{m} L_{0}\left(\underline{X}_{n-d_{1}-1, n}\right)}\right)\left(1-\Pi_{n}\right) \text {. }
$$

This proves the lemma.

LEMMA 3.4. The process $\left\{\eta_{n}\right\}_{n \geq d_{1}+1}$, where $\eta_{n}=\left(\underline{X}_{n-d_{1}-1, n}, \Pi_{n}\right)$, is a random Markov function. 
Proof. According to [12, Lemma 17, pp. 102-103] it is sufficient to show that $\eta_{n+1}$ is a function of the previous stage $\eta_{n}$ and of the variable $X_{n+1}$, and moreover the conditional distribution of $X_{n+1}$ given $\mathcal{F}_{n}$ is a function of $\eta_{n}$. Let us consider, for $x_{0}, \ldots, x_{d_{1}+2} \in \mathbb{E}, \alpha \in(0,1)$, the function

$$
\varphi\left(\underline{x}_{0, d_{1}+1}, \alpha, x_{d_{1}+2}\right)=\left(\underline{x}_{1, d_{1}+2}, \frac{f_{x_{d_{1}+1}}^{1}\left(x_{d_{1}+2}\right)(q+p \alpha)}{G_{1}\left(\underline{x}_{d_{1}+1, d_{1}+2}, \alpha\right)}\right) .
$$

We will show that $\eta_{n+1}=\varphi\left(\eta_{n}, X_{n+1}\right)$. Notice that (see Lemma A.5 or [9, Lemma A5] for $l=0$ )

$$
\Pi_{n+1}=\frac{f_{X_{n}}^{1}\left(X_{n+1}\right)\left(q+p \Pi_{n}\right)}{G_{1}\left(\underline{X}_{n, n+1}, \Pi_{n}\right)} .
$$

Hence

$$
\begin{aligned}
\varphi\left(\eta_{n}, X_{n+1}\right) & =\varphi\left(\underline{X}_{n-d_{1}-1, n}, \Pi_{n}, X_{n+1}\right) \\
& =\left(\underline{X}_{n-d_{1}, n}, X_{n+1}, \frac{f_{X_{n}}^{1}\left(X_{n+1}\right)\left(q+p \Pi_{n}\right)}{G_{1}\left(\underline{X}_{n, n+1}, \Pi_{n}\right)}\right) \\
& =\left(\underline{X}_{n-d_{1}, n+1}, \Pi_{n+1}\right)=\eta_{n+1} .
\end{aligned}
$$

Define $\hat{\mathcal{F}}_{n}=\sigma\left(\theta, \underline{X}_{0, n}\right)$. To see that the conditional distribution of $X_{n+1}$ given $\mathcal{F}_{n}$ is a function of $\eta_{n}$, let us consider the conditional expectation of $u\left(X_{n+1}\right)$ for any Borel function $u: \mathbb{E} \rightarrow \mathbb{R}$ given $\mathcal{F}_{n}$. The properties of the conditional expectation and Lemma A.1 imply

$$
\begin{aligned}
\mathbf{E}_{x}\left(u\left(X_{n+1}\right) \mid \mathcal{F}_{n}\right)= & \mathbf{E}_{x}\left(u\left(X_{n+1}\right)\left(1-\Pi_{n+1}\right) \mid \mathcal{F}_{n}\right)+\mathbf{E}_{x}\left(u\left(X_{n+1}\right) \Pi_{n+1} \mid \mathcal{F}_{n}\right) \\
= & \mathbf{E}_{x}\left(u\left(X_{n+1}\right) \mathbb{1}_{\{\theta>n+1\}} \mid \mathcal{F}_{n}\right)+\mathbf{E}_{x}\left(u\left(X_{n+1}\right) \mathbb{1}_{\{\theta \leq n+1\}} \mid \mathcal{F}_{n}\right) \\
= & \mathbf{E}_{x}\left(\mathbf{E}_{x}\left(u\left(X_{n+1}\right) \mathbb{1}_{\{\theta>n+1\}} \mid \hat{\mathcal{F}}_{n}\right) \mid \mathcal{F}_{n}\right) \\
& +\mathbf{E}_{x}\left(\mathbf{E}_{x}\left(u\left(X_{n+1}\right) \mathbb{1}_{\{\theta \leq n+1\}} \mid \hat{\mathcal{F}}_{n}\right) \mid \mathcal{F}_{n}\right) \\
= & \mathbf{E}_{x}\left(\mathbb{1}_{\{\theta>n+1\}} \mathbf{E}_{x}\left(u\left(X_{n+1}\right) \mid \hat{\mathcal{F}}_{n}\right) \mid \mathcal{F}_{n}\right) \\
& +\mathbf{E}_{x}\left(\mathbb{1}_{\{\theta \leq n+1\}} \mathbf{E}_{x}\left(u\left(X_{n+1}\right) \mid \hat{\mathcal{F}}_{n}\right) \mid \mathcal{F}_{n}\right) \\
= & \int_{\mathbb{E}} u(y) f_{X_{n}}^{0}(y) \mu(d y) \mathbf{P}_{x}\left(\theta>n+1 \mid \mathcal{F}_{n}\right) \\
& +\int_{\mathbb{E}} u(y) f_{X_{n}}^{1}(y) \mu(d y) \mathbf{P}_{x}\left(\theta \leq n+1 \mid \mathcal{F}_{n}\right) \\
= & \int_{\mathbb{E}} u(y)\left(p\left(1-\Pi_{n}\right) f_{X_{n}}^{0}(y)+\left(q+p \Pi_{n}\right) f_{X_{n}}^{1}(y)\right) \mu(d y) \\
= & \int_{\mathbb{E}} u(y) G_{1}\left(X_{n}, y, \Pi_{n}\right) \mu(d y) .
\end{aligned}
$$

This is our claim. 
Lemmata 3.3 and 3.4 are crucial for the solution of the problem 2.5. They show that the initial problem can be reduced to the problem of stopping the Markov random function $\eta_{n}=\left(\underline{X}_{n-d_{1}-1, n}, \Pi_{n}\right)$ with the payoff given by $(3.2)$. As a consequence, we can use the tools of optimal stopping theory to find the stopping time $\tau^{*}$ such that

$$
\mathbf{E}_{x}\left[h\left(\underline{X}_{\tau^{*}-d_{1}-1, \tau^{*}}, \Pi_{\tau^{*}}\right)\right]=\sup _{\tau \in \mathfrak{S}_{d_{1}+1}^{X}} \mathbf{E}_{x}\left[h\left(\underline{X}_{\tau-d_{1}-1, \tau}, \Pi_{\tau}\right)\right] .
$$

To solve (3.4), for any Borel function $u: \mathbb{E}^{d_{1}+2} \times[0,1] \rightarrow \mathbb{R}$ define

$$
\begin{aligned}
& \mathbf{T} u\left(\underline{x}_{0, d_{1}+1}, \alpha\right)=\mathbf{E}_{x}\left[u\left(\underline{X}_{n-d_{1}, n+1}, \Pi_{n+1}\right) \mid \underline{X}_{n-1-d_{1}, n}=\underline{x}_{0, d_{1}+1}, \Pi_{n}=\alpha\right], \\
& \mathbf{Q} u\left(\underline{x}_{0, d_{1}+1}, \alpha\right)=\max \left\{u\left(\underline{x}_{0, d_{1}+1}, \alpha\right), \mathbf{T} u\left(\underline{x}_{0, d_{1}+1}, \alpha\right)\right\} .
\end{aligned}
$$

By the definition of $\mathbf{T}$ and $\mathbf{Q}$ we get

Lemma 3.5. For the payoff function $h\left(\underline{x}_{0, d_{1}+1}, \alpha\right)$ characterized by 3.2 and for the sequence $\left\{r_{k}\right\}_{k=0}^{\infty}$ given by

$$
\begin{aligned}
r_{0}\left(\underline{x}_{1, d_{1}+1}\right)=p\left[1-p^{d_{2}}+q \sum_{m=0}^{d_{1}} \frac{L_{m-1}\left(\underline{x}_{1, d_{1}+1}\right)}{p^{m} L_{0}\left(\underline{x}_{1, d_{1}+1}\right)}\right] \\
r_{k}\left(\underline{x}_{1, d_{1}+1}\right)=p \int_{\mathbb{E}} \max \left\{1-p^{d_{2}}+q \sum_{m=1}^{d_{1}+1} \frac{L_{m}\left(\underline{x}_{1, d_{1}+2}\right)}{p^{m} L_{0}\left(\underline{x}_{1, d_{1}+2}\right)} ; r_{k-1}\left(\underline{x}_{2, d_{1}+2}\right)\right\} \\
\quad \times f_{x_{d_{1}+1}}^{0}\left(x_{d_{1}+2}\right) \mu\left(d x_{d_{1}+2}\right),
\end{aligned}
$$

the following formulae hold:

$$
\mathbf{Q}^{k} h\left(\underline{x}_{1, d_{1}+2}, \alpha\right)
$$

$$
=(1-\alpha) \max \left\{1-p^{d_{2}}+q \sum_{m=1}^{d_{1}+1} \frac{L_{m}\left(\underline{x}_{1, d_{1}+2}\right)}{p^{m} L_{0}\left(\underline{x}_{1, d_{1}+2}\right)} ; r_{k-1}\left(\underline{x}_{2, d_{1}+2}\right)\right\}, \quad k \geq 1,
$$

$\mathbf{T Q}^{k} h\left(\underline{x}_{1, d_{1}+2}, \alpha\right)=(1-\alpha) r_{k}\left(\underline{x}_{2, d_{1}+2}\right), \quad k \geq 0$.

Proof. By the definition of the operator $\mathbf{T}$ and using Lemma A.5 (with $l=0)$ given that $\left(\underline{X}_{n-d_{1}-1, n}, \Pi_{n}\right)=\left(\underline{x}_{1, d_{1}+2}, \alpha\right)$ we get

$$
\begin{gathered}
\mathbf{T} h\left(\underline{x}_{1, d_{1}+2}, \alpha\right)=\mathbf{E}_{x}\left[h\left(\underline{X}_{n-d_{1}, n+1}, \Pi_{n+1}\right) \mid \underline{X}_{n-d_{1}-1, n}=\underline{x}_{1, d_{1}+2}, \Pi_{n}=\alpha\right] \\
=\mathbf{E}_{x}\left[\left(1-p^{d_{2}}+q \sum_{m=1}^{d_{1}+1} \frac{L_{m}\left(\underline{X}_{n-d_{1}, n+1}\right)}{p^{m} \underline{x}_{0}\left(\underline{\underline{X}}_{n-d_{1}, n+1}\right)}\right)\left(1-\Pi_{n+1}\right) \mid \underline{X}_{n-d_{1}-1, n}=\underline{x}_{1, d_{1}+2}, \Pi_{n}=\alpha\right] \\
=p(1-\alpha) \int\left(1-p_{\mathbb{E}}^{d_{2}}+q \sum_{m=1}^{d_{1}+1} \frac{L_{m-1}\left(\underline{x}_{2, d_{1}+2}\right)}{p^{m} L_{0}\left(\underline{x}_{2, d_{1}+2}\right)} \frac{f_{x_{d_{1}+2}}^{1}\left(x_{d_{1}+3}\right)}{f_{x_{d_{1}+2}}^{0}\left(x_{d_{1}+3}\right)}\right) \\
\times \frac{f_{x_{d_{1}+2}}^{0}\left(x_{d_{1}+3}\right) G_{1}\left(\underline{x}_{d_{1}+2, d_{1}+3}, \alpha\right)}{G_{1}\left(\underline{x}_{d_{1}+2, d_{1}+3}, \alpha\right)} \mu\left(d x_{d_{1}+3}\right)
\end{gathered}
$$




$$
\begin{aligned}
& =p(1-\alpha)\left[1-p^{d_{2}}+q \sum_{m=1}^{d_{1}+1} \int_{\mathbb{E}} \frac{L_{m-1}\left(\underline{x}_{2, d_{1}+2}\right)}{p^{m} L_{0}\left(\underline{x}_{2, d_{1}+2}\right)} f_{x_{d_{1}+2}}^{1}\left(x_{d_{1}+3}\right) \mu\left(d x_{d_{1}+3}\right)\right] \\
& =(1-\alpha) p\left[1-p^{d_{2}}+q \sum_{m=1}^{d_{1}+1} \frac{L_{m-1}\left(\underline{x}_{2, d_{1}+2}\right)}{p^{m} L_{0}\left(\underline{x}_{2, d_{1}+2}\right)}\right]=(1-\alpha) r_{0}\left(\underline{x}_{2, d_{1}+2}\right) .
\end{aligned}
$$

Directly from the definition of $\mathbf{Q}$ we get

$$
\begin{aligned}
\mathbf{Q} h\left(\underline{x}_{1, d_{1}+2}, \alpha\right) & =\max \left\{h\left(\underline{x}_{1, d_{1}+2}, \alpha\right) ; \mathbf{T} h\left(\underline{x}_{1, d_{1}+2}, \alpha\right)\right\} \\
& =(1-\alpha) \max \left\{1-p^{d_{2}}+q \sum_{m=1}^{d_{1}+1} \frac{L_{m}\left(\underline{x}_{1, d_{1}+2}\right)}{p^{m} L_{0}\left(\underline{x}_{1, d_{1}+2}\right)} ; r_{0}\left(\underline{x}_{2, d_{1}+2}\right)\right\} .
\end{aligned}
$$

Suppose now that Lemma 3.5 holds for $\mathbf{T} \mathbf{Q}^{k-1} h$ and $\mathbf{Q}^{k} h$ for some $k>1$. Then, using a similar transformation to the case of $k=0$, we get

$$
\begin{aligned}
\mathbf{T Q}^{k} h\left(\underline{x}_{1, d_{1}+2}, \alpha\right) & \\
= & \mathbf{E}_{x}\left[\mathbf{Q}^{k} h\left(\underline{X}_{n-d_{1}, n+1}, \Pi_{n+1}\right) \mid \underline{X}_{n-d_{1}-1, n}=\underline{x}_{1, d_{1}+2}, \Pi_{n}=\alpha\right] \\
= & \int_{\mathbb{E}}\left[\max \left\{1-p^{d_{2}}+q \sum_{m=1}^{d_{1}+1} \frac{L_{m}\left(\underline{x}_{2, d_{1}+3}\right)}{p^{m} L_{0}\left(\underline{x}_{2, d_{1}+3}\right)} ; r_{k-1}\left(\underline{x}_{3, d_{1}+3}\right)\right\}\right. \\
& \left.\times(1-\alpha) p f_{x_{d_{1}+2}}^{0}\left(x_{d_{1}+3}\right)\right] \mu\left(d x_{d_{1}+3}\right) \\
= & (1-\alpha) r_{k}\left(\underline{x}_{2, d_{1}+2}\right) .
\end{aligned}
$$

Moreover,

$$
\begin{aligned}
\mathbf{Q}^{k+1} h\left(\underline{x}_{1, d_{1}+2}, \alpha\right) & =\max \left\{h\left(\underline{x}_{1, d_{1}+2}, \alpha\right) ; \mathbf{T} \mathbf{Q}^{k} h\left(\underline{x}_{1, d_{1}+2}, \alpha\right)\right\} \\
= & (1-\alpha) \max \left\{1-p^{d_{2}}+q \sum_{m=1}^{d_{1}+1} \frac{L_{m}\left(\underline{x}_{1, d_{1}+2}\right)}{p^{m} L_{0}\left(\underline{x}_{1, d_{1}+2}\right)} ; r_{k}\left(\underline{x}_{2, d_{1}+2}\right)\right\} .
\end{aligned}
$$

This completes the proof.

The following theorem is the main result of the paper.

THEOREM 3.6.

(a) The solution of the problem (2.5) is given by

$$
\tau^{*}=\inf \left\{n \geq d_{1}+1: 1-p^{d_{2}}+q \sum_{m=1}^{d_{1}+1} \frac{L_{m}\left(\underline{X}_{n-d_{1}-1, n}\right)}{p^{m} L_{0}\left(\underline{X}_{n-d_{1}-1, n}\right)} \geq r^{*}\left(\underline{X}_{n-d_{1}, n}\right)\right\}
$$

where $r^{*}\left(\underline{X}_{n-d, n}\right)=\lim _{k \rightarrow \infty} r_{k}\left(\underline{X}_{n-d, n}\right)$.

(b) The value of the problem, i.e. the maximal probability for 2.5 given $X_{0}=x$, is equal to 


$$
\begin{array}{r}
\mathbf{P}_{x}\left(-d_{1} \leq \theta-\tau^{*} \leq d_{2}\right) \\
=(1-\pi) p^{d_{1}+1} \int_{\mathbb{E}^{d_{1}+1}} \max \left\{1-p^{d_{2}}+q \sum_{\substack{m=1 \\
d_{1}+1}} \frac{L_{m}\left(x, \underline{x}_{1, d_{1}+1}\right)}{p^{m} L_{0}\left(x, \underline{x}_{1, d_{1}+1}\right)} ; r^{*}\left(\underline{x}_{1, d_{1}+1}\right)\right\} \\
\times L_{0}\left(x, \underline{x}_{1, d_{1}+1}\right) \mu\left(d x_{1}, \ldots, d x_{d_{1}+1}\right) .
\end{array}
$$

Proof. (a) According to Lemma 3.2 we look for a stopping time equal at least $d_{1}+1$. From optimal stopping theory [12] we know that $\tau_{0}$ defined by (3.1) can be expressed as

$$
\tau_{0}=\inf \left\{n \geq d_{1}+1: h\left(\underline{X}_{n-1-d_{1}, n}, \Pi_{n}\right) \geq \mathbf{Q}^{*} h\left(\underline{X}_{n-1-d_{1}, n}, \Pi_{n}\right)\right\}
$$

where $\mathbf{Q}^{*} h\left(\underline{X}_{n-1-d_{1}, n}, \Pi_{n}\right)=\lim _{k \longrightarrow \infty} \mathbf{Q}^{k} h\left(\underline{X}_{n-1-d_{1}, n}, \Pi_{n}\right)$. According to Lemma 3.5 ,

$$
\begin{aligned}
\tau_{0}= & \inf \left\{n \geq d_{1}+1: 1-p^{d_{2}}+q \sum_{m=1}^{d_{1}+1} \frac{L_{m}\left(\underline{X}_{n-d_{1}-1, n}\right)}{p^{m} L_{0}\left(\underline{X}_{n-d_{1}-1, n}\right)}\right. \\
& \left.\geq \max \left\{1-p^{d-2}+q \sum_{m=1}^{d_{1}+1} \frac{L_{m}\left(\underline{X}_{n-d_{1}-1, n}\right)}{p^{m} L_{0}\left(\underline{X}_{n-d_{1}-1, n}\right)} ; r^{*}\left(\underline{X}_{n-d_{1}, n}\right)\right\}\right\} \\
= & \inf \left\{n \geq d_{1}+1: 1-p^{d_{2}}+q \sum_{m=1}^{d_{1}+1} \frac{L_{m}\left(\underline{X}_{n-d_{1}-1, n}\right)}{p^{m} L_{0}\left(\underline{X}_{n-d_{1}-1, n}\right)} \geq r^{*}\left(\underline{X}_{n-d_{1}, n}\right)\right\}=\tau^{*} .
\end{aligned}
$$

(b) By known facts from optimal stopping theory we can write

$$
\begin{aligned}
& \mathbf{P}_{x}\left(-d_{1} \leq \theta-\tau^{*} \leq d_{2}\right)=\mathbf{E}_{x}\left(Q^{*} h\left(\underline{X}_{0, d_{1}+1}, \Pi_{d_{1}+1}\right)\right) \\
& =\mathbf{E}_{x}\left(\left(1-\Pi_{d_{1}+1}\right) \max \left\{1-p^{d_{2}}+q \sum_{m=1}^{d_{1}+1} \frac{L_{m}\left(\underline{X}_{0, d_{1}+1}\right)}{p^{m} L_{0}\left(\underline{X}_{0, d_{1}+1}\right)} ; r^{*}\left(\underline{X}_{1, d_{1}+1}\right)\right\}\right) \\
& =\mathbf{E}_{x}\left(\mathbf{E}_{x}\left(\mathbb{1}_{\left\{\theta>d_{1}+1\right\}} \mid \mathcal{F}_{d_{1}+1}\right) \max \left\{1-p^{d_{2}}+q \sum_{m=1}^{d_{1}+1} \frac{L_{m}\left(\underline{X}_{0, d_{1}+1}\right)}{p^{m} L_{0}\left(\underline{X}_{0, d_{1}+1}\right)} ; r^{*}\left(\underline{X}_{1, d_{1}+1}\right)\right\}\right) \\
& =\mathbf{E}_{x}\left(\mathbb{1}_{\left\{\theta>d_{1}+1\right\}} \max \left\{1-p^{d_{2}}+q \sum_{m=1}^{d_{1}+1} \frac{L_{m}\left(\underline{X}_{0, d_{1}+1}\right)}{p^{m} L_{0}\left(\underline{X}_{0, d_{1}+1}\right)} ; r^{*}\left(\underline{X}_{1, d_{1}+1}\right)\right\}\right) \\
& =\mathbf{P}_{x}\left(\theta>d_{1}+1\right) \int \max \left\{1-p^{d_{2}}+q \sum_{\mathbb{E}^{d_{1}+1}}^{d_{1}+1} \frac{L_{m}\left(x, \underline{x}_{1, d_{1}+1}\right)}{p^{m} L_{0}\left(x, \underline{x}_{1, d_{1}+1}\right)} ; r^{*}\left(\underline{x}_{1, d_{1}+1}\right)\right\} \\
& \quad \times L_{0}\left(x, \underline{x}_{1, d_{1}+1}\right) \mu\left(d x_{1}, \ldots, d x_{d_{1}+1}\right) .
\end{aligned}
$$

This ends the proof of the theorem.

Final remarks. The analysis we have presented concerns the case when the acceptable error for detecting the switching time is different for underes- 
timating and overestimating. In other words, we can stop the observation $d_{1}$ units of time before the switching time, but no later than $d_{2}$ units after that time. If we admit that the sequence under observation may be homogeneous, that is, without any switching time, it is important for the observer to have a criterion to decide that the sequence observed is entirely of the second type (that is, there were no data of the type "before switching"). This can be verified by the standard testing procedure when we stop very early (at $\tau \leq \min \left\{d_{1}, d_{2}\right\}$.

\section{Appendix. Lemmata}

Lemma A.1. Let $n>0$ and $k \geq 0$. Then

$$
\mathbf{P}_{x}\left(\theta \leq n+k \mid \mathcal{F}_{n}\right)=1-p^{k}\left(1-\Pi_{n}\right) .
$$

Proof. It is sufficient to show that for $D \in \mathcal{F}_{n}$,

$$
\int_{D} \mathbb{1}_{\{\theta>n+k\}} d \mathbf{P}_{x}=\int_{D} p^{k}\left(1-\Pi_{n}\right) d \mathbf{P}_{x} .
$$

Define $\widetilde{\mathcal{F}}_{n}=\sigma\left(\mathcal{F}_{n}, \mathbb{1}_{\{\theta>n\}}\right)$. We have

$$
\begin{aligned}
\int_{D} \mathbb{1}_{\{\theta>n+k\}} d \mathbf{P}_{x} & =\int_{D} \mathbb{1}_{\{\theta>n+k\}} \mathbb{1}_{\{\theta>n\}} d \mathbf{P}_{x}=\int_{D \cap\{\theta>n\}} \mathbb{1}_{\{\theta>n+k\}} d \mathbf{P}_{x} \\
& =\int_{D \cap\{\theta>n\}} \mathbf{E}_{x}\left(\mathbb{1}_{\{\theta>n+k\}} \mid \widetilde{\mathcal{F}}_{n}\right) d \mathbf{P}_{x} \\
& =\int_{D \cap\{\theta>n\}} \mathbf{E}_{x}\left(\mathbb{1}_{\{\theta>n+k\}} \mid \theta>n\right) d \mathbf{P}_{x} \\
& =\int_{D} \mathbb{1}_{\{\theta>n\}} p^{k} d \mathbf{P}_{x}=\int_{D}\left(1-\Pi_{n}\right) p^{k} d \mathbf{P}_{x} .
\end{aligned}
$$

Lemma A.2. For $n>0$,

$$
\mathbf{P}_{x}\left(\theta>n \mid \mathcal{F}_{n}\right)=1-\Pi_{n}=\frac{\bar{\pi} p^{n} L_{0}\left(\underline{X}_{0, n}\right)}{S_{n}\left(\underline{X}_{0, n}\right)} .
$$

Proof. Set $\underline{D}_{0, n}=\left\{\omega: \underline{X}_{0, n} \in \underline{A}_{0, n}, A_{i} \in \mathcal{B}\right\}$. Then

$$
\begin{aligned}
\mathbf{P}_{x}\left(\underline{D}_{0, n}\right) \mathbf{P}_{x}(\theta & \left.>n \mid \underline{D}_{0, n}\right)=\int_{\underline{D}_{0, n}} \mathbb{1}_{\{\theta>n\}} d \mathbf{P}_{x}=\int_{\underline{D}_{0, n}} \mathbf{P}_{x}\left(\theta>n \mid \mathcal{F}_{n}\right) d \mathbf{P}_{x} \\
& =\int_{\underline{A}_{0, n}} \frac{\bar{\pi} p^{n} L_{0}\left(\underline{x}_{0, n}\right)}{S_{n}\left(\underline{x}_{0, n}\right)} S_{n}\left(\underline{x}_{0, n}\right) \mu\left(d \underline{x}_{0, n}\right)=\int_{\underline{D}_{0, n}} \frac{\bar{\pi} p^{n} L_{0}\left(\underline{X}_{0, n}\right)}{S_{n}\left(\underline{X}_{0, n}\right)} d \mathbf{P}_{x} .
\end{aligned}
$$

Hence, by the definition of the conditional expectation, we get the assertion. 
Lemma A.3. For $\underline{x}_{0, l+1} \in \mathbb{E}^{l+2}, \alpha \in[0,1]$ and the functions $S .(\cdot)$ and $G$.(.) given by (2.3) and (2.4) we have

$$
S_{n}\left(\underline{X}_{0, n}\right)=S_{n-l-1}\left(\underline{X}_{0, n-l-1}\right) G_{l+1}\left(\underline{X}_{n-l-1, n}, \Pi_{n-l-1}\right) .
$$

Proof. By A.2 we have

$$
\begin{aligned}
& S_{n-l-1}\left(\underline{X}_{0, n-l-1}\right) \cdot G_{l+1}\left(\underline{X}_{n-l-1, n}, \Pi_{n-l-1}\right) \\
& =S_{n-l-1}\left(\underline{X}_{0, n-l-1}\right) \Pi_{n-l-1} L_{l+1}\left(\underline{X}_{n-l-1, n}\right)+S_{n-l-1}\left(\underline{X}_{0, n-l-1}\right)\left(1-\Pi_{n-l-1}\right) \\
& \times\left(\sum_{k=0}^{l} p^{l-k} q L_{k+1}\left(\underline{X}_{n-l-1, n}\right)+p^{l+1} L_{0}\left(\underline{X}_{n-l-1, n}\right)\right) \\
& \stackrel{\text { A.2 }}{=}\left(S_{n-l-1}\left(\underline{X}_{0, n-l-1}\right)-\bar{\pi} p^{n-l-1} L_{0}\left(\underline{X}_{0, n-l-1}\right)\right) L_{l+1}\left(\underline{X}_{n-l-1, n}\right) \\
& +\bar{\pi} p^{n-l-1} L_{0}\left(\underline{X}_{0, n-l-1}\right)\left(\sum_{k=0}^{l} p^{l-k} q L_{k+1}\left(\underline{X}_{n-l-1, n}\right)+p^{l+1} L_{0}\left(\underline{X}_{n-l-1, n}\right)\right) \\
& =\left(\pi L_{n-l-1}\left(\underline{X}_{0, n-l-1}\right)+\bar{\pi} \sum_{k=1}^{n-l-1} p^{k-1} q L_{n-k-l}\left(\underline{X}_{0, n-l-1}\right)\right) L_{l+1}\left(\underline{X}_{n-l-1, n}\right) \\
& +\bar{\pi}\left(\sum_{k=0}^{l} p^{n-k-1} q L_{k+1}\left(\underline{X}_{0, n}\right)+p^{n} L_{0}\left(\underline{X}_{0, n}\right)\right) .
\end{aligned}
$$

On the other hand,

$$
\begin{aligned}
& S_{n}\left(\underline{X}_{0, n}\right)=\pi L_{n}\left(\underline{X}_{0, n}\right)+\bar{\pi}\left(\sum_{k=1}^{n} p^{k-1} q L_{k}\left(\underline{X}_{0, n}\right)+p^{n} L_{0}\left(\underline{X}_{0, n}\right)\right) \\
& =\pi L_{n}\left(\underline{X}_{0, n}\right) \\
& +\bar{\pi}\left(\sum_{k=1}^{n-l-1} p^{k-1} q L_{n-k+1}\left(\underline{X}_{0, n}\right)+\sum_{k=n-l}^{n} p^{k-1} q L_{n-k+1}\left(\underline{X}_{0, n}\right)+p^{n} L_{0}\left(\underline{X}_{0, n}\right)\right) .
\end{aligned}
$$

This establishes A.3.

Lemma A.4. For $n>l \geq 0$,

$$
\mathbf{P}_{x}\left(\theta \leq n-l-1 \mid \mathcal{F}_{n}\right)=\frac{\Pi_{n-l-1} L_{l+1}\left(\underline{X}_{n-l-1, n}\right)}{G_{l+1}\left(\underline{X}_{n-l-1, n}, \Pi_{n-l-1}\right)} .
$$

Proof. Let $\underline{D}_{0, n}=\left\{\omega: \underline{X}_{0, n} \in \underline{A}_{0, n}, A_{i} \in \mathcal{B}\right\}$. Then

$$
\begin{aligned}
\mathbf{P}_{x}\left(\underline{D}_{0, n}\right) \mathbf{P}_{x}\left(\theta>n-l-1 \mid \underline{D}_{0, n}\right) & =\int_{\underline{D}_{0, n}} \mathbb{1}_{\{\theta>n-l-1\}} d \mathbf{P}_{x} \\
& =\int_{\underline{D}_{0, n}} \mathbf{P}_{x}\left(\theta>n-l-1 \mid \mathcal{F}_{n}\right) d \mathbf{P}_{x}
\end{aligned}
$$




$$
\begin{aligned}
= & \underline{A}_{0, n} \frac{\sum_{k=n-l}^{n} \mathbf{P}_{x}(\theta=k) L_{n-k+1}\left(\underline{x}_{0, n}\right)+\mathbf{P}_{x}(\theta>n) L_{0}\left(\underline{x}_{0, n}\right)}{S_{n}\left(\underline{x}_{0, n}\right)} S_{n}\left(\underline{x}_{0, n}\right) \mu\left(d \underline{x}_{0, n}\right) \\
= & \int_{\underline{A}_{0, n}} \frac{\bar{\pi} p^{n-l-1} L_{0}\left(\underline{x}_{0, n-l-1}\right)\left(\sum_{k=0}^{l} p^{l-k} q L_{k+1}\left(\underline{x}_{n-l-1, n}\right)+p^{l+1} L_{0}\left(\underline{x}_{n-l-1, n}\right)\right)}{S_{n}\left(\underline{x}_{0, n}\right)} \times S_{n}\left(\underline{x}_{0, n}\right) \mu\left(d \underline{x}_{0, n}\right) \\
= & \int_{0, n} \frac{\bar{\pi} p^{n-l-1} L_{0}\left(\underline{x}_{0, n-l-1}\right)\left(\sum_{k=0}^{l} p^{l-k} q L_{k+1}\left(\underline{X}_{n-l-1, n}\right)+p^{l+1} L_{0}\left(\underline{X}_{n-l-1, n}\right)\right)}{S\left(\underline{X}_{0, n}\right)} d \mathbf{P}_{x} \\
& \stackrel{\overline{\mathrm{A} .3}}{=} \int_{\underline{D}_{0, n}} \frac{\bar{\pi} p^{n-l-1} L_{0}\left(\underline{x}_{0, n-l-1}\right)\left(\sum_{k=0}^{l} p^{l-k} q L_{k+1}\left(\underline{X}_{n-l-1, n}\right)+p^{l+1} L_{0}\left(\underline{X}_{n-l-1, n}\right)\right)}{S_{n-l-1}\left(\underline{X}_{0, n-l-1}\right) G_{l+1}\left(\underline{X}_{n-l-1, n}, \Pi_{n-l-1}\right)} d \mathbf{P}_{x} \\
& \int_{\underline{D}_{0, n}}\left(1-\Pi_{n-l-1}\right) \frac{\sum_{k=0}^{l} p^{l-k} q L_{k+1}\left(\underline{X}_{n-l-1, n}\right)+p^{l+1} L_{0}\left(\underline{X}_{n-l-1, n}\right)}{G_{l+1}\left(\underline{X}_{n-l-1, n}, \Pi \Pi_{n-l-1}\right)} d \mathbf{P}_{x} .
\end{aligned}
$$

This implies that

$$
\begin{aligned}
& \mathbf{P}_{x}\left(\theta>n-l-1 \mid \mathcal{F}_{n}\right) \\
& \quad=\left(1-\Pi_{n-l-1}\right) \frac{\sum_{k=0}^{l} p^{l-k} q L_{k+1}\left(\underline{X}_{n-l-1, n}\right)+p^{l+1} L_{0}\left(\underline{X}_{n-l-1, n}\right)}{G_{l+1}\left(\underline{X}_{n-l-1, n}, \Pi_{n-l-1}\right)} .
\end{aligned}
$$

Simple transformations of A.4 lead to the assertion.

LEMMA A.5. For $n>l \geq 0$ the following recursive equation holds:

$$
\Pi_{n}=\frac{\Pi_{n-l-1} L_{l+1}\left(\underline{X}_{n-l-1, n}\right)+\left(1-\Pi_{n-l-1}\right) q \sum_{k=0}^{l} p^{l-k} L_{k+1}\left(\underline{X}_{n-l-1, n}\right)}{G_{l+1}\left(\underline{X}_{n-l-1, n}, \Pi_{n-l-1}\right)} .
$$

Proof. With the aid of A.2 we get

$$
\frac{1-\Pi_{n}}{1-\Pi_{n-l-1}}=\frac{p^{n} L_{0}\left(\underline{X}_{0, n}\right)}{S_{n}\left(\underline{X}_{0, n}\right)} \frac{S_{n}\left(\underline{X}_{0, n-l-1}\right)}{p^{n-l-1} L_{0}\left(\underline{X}_{0, n-l-1}\right)}=\frac{p^{l+1} L_{0}\left(\underline{X}_{n-l-1, n}\right)}{G_{l+1}\left(\underline{X}_{n-l-1, n}, \Pi_{n-l-1}\right)} .
$$

Hence

$$
\begin{aligned}
\Pi_{n} & =\frac{G_{l+1}\left(\underline{X}_{n-l-1, n}, \Pi_{n-l-1}\right)-p^{n-l-1} L_{0}\left(\underline{X}_{0, n-l-1}\right)\left(1-\Pi_{n-l-1}\right)}{G_{l+1}\left(\underline{X}_{n-l-1, n}, \Pi_{n-l-1}\right)} \\
& =\frac{\Pi_{n-l-1} L_{l+1}\left(\underline{X}_{n-l-1, n}\right)+\left(1-\Pi_{n-l-1}\right) q \sum_{k=0}^{l} p^{l-k} L_{k+1}\left(\underline{X}_{n-l-1, n}\right)}{G_{l+1}\left(\underline{X}_{n-l-1, n}, \Pi_{n-l-1}\right)}
\end{aligned}
$$

Acknowledgements. The authors are pleased to thank the anonymous referee for many useful comments and improvements. 
This research was partly supported by Wrocław University of Technology (grant nos. S50099/K1110 and S20096).

\section{References}

[1] M. Baron, Early detection of epidemics as a sequential change-point problem, in: V. Antonov et al. (eds.), Longevity, Aging and Degradation Models in Reliability, Public Health, Medicine and Biology, LAD 2004 (St. Petersburg, 2004), IMS Lecture Notes Monogr. Ser. 2, St. Petersburg State Politechnical Univ., 2004, 31-43.

[2] M. Basseville and A. Benveniste (eds.), Detection of Abrupt Changes in Signals and Dynamical Systems, Lecture Notes in Control and Information Sci. 77, Springer, Berlin, 1986.

[3] T. Bojdecki, Probability maximizing approach to optimal stopping and its application to a disorder problem, Stochastics 3 (1979), 61-71.

[4] T. Bojdecki and J. Hosza, On a generalized disorder problem, Stochastic Processes Appl. 18 (1984), 349-359.

[5] B. E. Brodsky and B. S. Darkhovsky, Nonparametric Methods in Change-Point Problems, Math. Appl. 243, Kluwer, Dordrecht, 1993.

[6] P. Dube and R. Mazumdar, A framework for quickest detection of traffic anomalies in networks, technical report, Electrical and Computer Engineering, Purdue Univ., 2001; citeseer.ist.psu.edu/506551.html.

[7] G. V. Moustakides, Quickest detection of abrupt changes for a class of random processes, IEEE Trans. Information Theory 44 (1998), 1965-1968.

[8] A. Ochman-Gozdek and K. Szajowski, Detection of a random sequence of disorders, in: X. He (ed.), Proc. 59th ISI World Statistics Congress (Hong Kong, 2013), 37953800; http://2013.isiproceedings.org/Files/CPS018-P8-S.pdf.

[9] W. Sarnowski and K. Szajowski, Optimal detection of transition probability change in random sequence, Stochastics 83 (2011), 569-581.

[10] W. A. Shewhart, Economic Control of Quality of Manufactured Products, Van Nostrand, New York, 1931.

[11] A. N. Shiryaev, The detection of spontaneous effects, Soviet Math. Dokl. 2 (1961), 740-743; translation from Dokl. Akad. Nauk SSSR 138 (1961), 799-801.

[12] A. N. Shiryaev, Optimal Stopping Rules, Springer, New York, 1978.

[13] D. Siegmund, Change-points: from sequential detection to biology and back, Sequential Anal. 32 (2013), 2-14.

[14] K. Szajowski, Optimal on-line detection of outside observation, J. Statist. Planning Inference 30 (1992), 413-426.

[15] K. Szajowski, A two-disorder detection problem, Appl. Math. (Warsaw) 24 (1996), $231-241$.

[16] A. G. Tartakovsky, B. L. Rozovskii, R. B. Blažek, and H. Kim, Detection of intrusions in information systems by sequential change-point methods, Statist. Methodol. 3 (2006), 252-293.

[17] B. Yakir, Optimal detection of a change in distribution when the observations form a Markov chain with a finite state space, in: E. Carlstein et al. (eds.), Change-Point Problems (South Hadley, MA, 1992), IMS Lecture Notes Monogr. Ser. 23, Inst. Math. Statist., Hayward, CA, 1994, 346-358.

[18] M. Yoshida, Probability maximizing approach for a quickest detection problem with complicated Markov chain, J. Information Optim. Sci. 4 (1983), 127-145. 
Aleksandra Ochman-Gozdek

Objectivity Bespoke Software Specialists Ltd.

Strzegomska 142a

54-429 Wrocław, Poland

E-mail: aochman@o2.pl

Krzysztof Szajowski

Faculty of Pure and Applied Mathematics

Wrocław University of Science and Technology

Wybrzeże Wyspiańskiego 27

50-370 Wrocław, Poland

E-mail: Krzysztof.Szajowski@pwr.edu.pl
Wojciech Sarnowski Business Intelligence Team at Krajowy Rejestr Długów Armii Ludowej 21 51-214 Wrocław, Poland E-mail: sarnowsk@interia.pl 